\title{
Experience in community child health as part of training in child and adolescent psychiatry
}

\author{
Peter Hill, Senior Lecturer in Child and Adolescent Psychiatry, St George's Hospital \\ Medical School; JUdITH DawkINs, Registrar in Psychiatry, St George's Hospital; \\ Mary AnNe Griffiths, Consultant in Child and Adolescent Psychiatry, Chertsey \\ Child Guidance Clinic; and RUTH TALBOT, Senior Registrar in Child and Adolescent \\ Psychiatry, St George's Hospital, Tooting, London SW17
}

Careers in psychiatry which commence straight after house jobs are now commonplace and an informal tradition by which future child psychiatrists worked in paediatrics before training in psychiatry is nowadays less rehearsed. Recently, one of the St George's psychiatric registrar rotations arranged a six month post as a community child health doctor (community clinical medical officer) for a psychiatric registrar (JD) who had expressed an interest in child psychiatry as a career but lacked experience in paediatrics.

We examine the contribution of experience in community child health to training in child and adolescent psychiatry in the light of the registrar's experience adding the perspectives of a consultant and senior registrar in child psychiatry who had also previously worked in community child health.

Community child health doctors are the contemporary descendents of the old 'school doctors'. They are not training posts for consultant status but exist in two grades: the senior (SCMO) and the 'ordinary' clinical medical officer (CMO), the latter being roughly comparable with a registrar. Most are women and work office hours in school-based clinics, child development clinics, special community clinics such as those for immunisation or enuresis, and conduct special medical examinations for non-accidental injury and reception into care, all in a community setting. The work is intensely clinical with large numbers of children being seen, many of them normal. We would like to detail the advantages of such experience so far as future child psychiatrists are concerned.

Familiarity with the normal range of child behaviour and development

For many child psychiatry trainees, experience of normal children is very limited. This places them at a disadvantage when asked to comment upon whether a given behaviour or emotional response is within normal limits. Community paediatric experience with large numbers of normal children enables doctors to become familiar with the progress and vicissitudes of normal psychological development and acquire the skill to form a quick global judgement as to a child's developmental, physical and mental health.

\section{The prognosis of minor variations in behaviour and development}

Experience is a better guide than textbooks as to whether certain behaviours and emotional responses will persist or attenuate with time. Most minor problems will not be referred to psychiatric services so that trainees within those services will not encounter them. Experience with a large number of normal or nearnormal children in community child health practice enables authoritative reassurance of concern to be expressed when appropriate in child psychiatry practice.

\section{Familiarity with the normal range of parenting and} parental reactions

Community child health doctors meet a large number of parents at varying stages of family development. It becomes easier to understand and appreciate common issues in parenting such as fatigue in the parents of young children, or the manner in which mid-life concerns interact with being a parent to adolescents.

\section{Interviewing and examining children and parents in} non-hospital settings

Many child psychiatrists will eventually work in community clinics. In community child health practice, the authority to take a child's clothes off or demand that a child undertakes some clinical task as part of the assessment cannot be derived from the hospital setting and has to be explained or negotiated. Good clinical habits, such as introducing oneself to children and explaining what one's role and purpose is, are thus learnt because they are necessary parts of the examination process.

\section{Handling skills for difiicult children}

When the above procedures of explanation and justification do not suffice to obtain a child's cooperation, persuasion and reassurance must be 
employed. Within community paediatrics, a number of procedures such as immunisation are viewed apprehensively by children. Experience with obtaining children's cooperation in such instances is valuable when in child psychiatric practice, it becomes necessary to get a young child to come into an interview room without the parents.

\section{Learning to establish credibility as a doctor outside hospitals}

It is not just children who question medical authority outside a hospital setting; the same applies to parents and other professionals involved with children's welfare. A scruffy presentation of oneself in a hospital setting may be tolerated but in a school setting professional authority would be diminished by overly casual dress and manner.

\section{Improving skills connected with physical aspects of children's medicine}

Familiarity with the physical examination of children as well as understanding how physical problems interact with educational progress provides a necessary dimension to child psychiatry which may be overlooked in psychiatric training.

\section{Counselling skills}

The work of a community child health doctor involves extensive counselling of adolescents at school. Because clinical medical officers do not prescribe, there is a necessary (rather than an elective) reliance on psychological methods of treatment.

\section{Gaining an outsider's perspective on mental health services}

Viewing the relevance, responsiveness and communicative aspects of specialist child mental health services from the outside can be salutary. Learning how to make a referral, persuade parents that it is necessary, and help them overcome anti-psychiatric prejudice is a further corrective experience to counterbalance an ivory tower perspective. It can be a revelation to learn that more than half of parents whose children have emotional and behaviour problems do not want a child psychiatry referral.

\section{Knowledge of the network of agencies}

Dealing with the services for child development problems and the various agencies which deal with the milder psychological problems of childhood facilitates later consultative work as a child psychiatrist.

\section{Knowledge of the educational system}

A similar point can be made about schools as institutions since clinical medical officers spend a large amount of their time in them. The range of attitudes of head teachers to pastoral care issues needs to be experienced in order to appreciate it. Experience of special education provisions and the assessment of children thought to require them is gained.

\section{Developing understanding of the work of health visitors and community child health doctors}

A self-evident but important aspect of the experience.

\section{Experience in working alone}

Clinical medical officers commonly work alone in their clinical settings. They learn how to maintain professional standards such as taking personal responsibility for continuing medical education, writing proper clinical notes, dealing with the tedium of repetitive clinical work, and maintaining credibility as children's doctors outside a hospital setting. Child psychiatrists frequently have to do the same in child guidance unit practice but may not experience working solo as doctors until they reach consultant level.

\section{Recommendations}

We think there are distinct advantages for future child and adolescent psychiatrists in obtaining experience in community child health. We consider that experience in community child health is more relevant than hospital paediatrics to future child and adolescent psychiatrists. In our view the experience should be for at least six months full-time. There would be advantages in a 12-month, part-time placement shared with child psychiatry because of the opportunities to follow certain children for longer. Registrar rotations might facilitate it as an option for those who wish to enter child psychiatry. Alternatively there is value in locating it as a more structured opportunity within higher training. In either case, arrangements would obviously need to be made with local community paediatric services who might see advantages in reciprocal arrangements for their own staff and trainees.

\section{Acknowledgements}

Thanks are due to Dr David Hall, Consultant Community Paediatrician, Wandsworth, for facilitating JD's attachment to his service and for his comments on this paper. 